\title{
The Procedural Index for Mortality Risk (PIMR): an index calculated using administrative data to quantify the independent influence of procedures on risk of hospital death
}

\author{
Carl van Walraven ${ }^{1,2,3^{*}}$, Jenna Wong ${ }^{1,2}$, Carol Bennett ${ }^{1,2}$ and Alan J Forster ${ }^{1,2,3}$
}

\begin{abstract}
Background: Surgeries and other procedures can influence the risk of death in hospital. All published scales that predict post-operative death risk require clinical data and cannot be measured using administrative data alone. This study derived and internally validated an index that can be calculated using administrative data to quantify the independent risk of hospital death after a procedure.
\end{abstract}

Methods: For all patients admitted to a single academic centre between 2004 and 2009, we estimated the risk of all-cause death using the Kaiser Permanente Inpatient Risk Adjustment Methodology (KP-IRAM). We determined whether each patient underwent one of 503 commonly performed therapeutic procedures using Canadian Classification of Interventions codes and whether each procedure was emergent or elective. Multivariate logistic regression modeling was used to measure the association of each procedure-urgency combination with death in hospital independent of the KP-IRAM risk of death. The final model was modified into a scoring system to quantify the independent influence each procedure had on the risk of death in hospital.

Results: 275460 hospitalizations were included (137,730 derivation, 137,730 validation). In the derivation group, the median expected risk of death was $0.1 \%$ (IQR 0.01\%-1.4\%) with 4013 (2.9\%) dying during the hospitalization. 56 distinct procedure-urgency combinations entered our final model resulting in a Procedural Index for Mortality Rating (PIMR) score values ranging from -7 to +11 . In the validation group, the PIMR score significantly predicted the risk of death by itself (c-statistic 67.3\%, 95\% Cl 66.6-68.0\%) and when added to the KP-IRAM model (c-index improved significantly from 0.929 to 0.938 ).

Conclusions: We derived and internally validated an index that uses administrative data to quantify the independent association of a broad range of therapeutic procedures with risk of death in hospital. This scale will improve risk adjustment when administrative data are used for analyses.

\section{Background}

Surgeries and procedures are major functions of hospitals that importantly influence patient outcomes and hospital performance. Procedural outcomes are often used to compare surgeons, clinical divisions, hospitals, and health jurisdictions. Many different types of surgeries and procedures exist in different specialties,

\footnotetext{
* Correspondence: carlv@ohri.ca

${ }^{1}$ Clinical Epidemiology Program, Ottawa Hospital Research Institute, 1053

Carling Avenue, Ottawa, K1Y 4E9, Canada

Full list of author information is available at the end of the article
}

involving very different patient populations. As a result, the influence of different types of procedures on hospital outcomes can vary greatly.

Quantifying the independent influence of a broad range of different types of procedures on outcomes would allow analysts, administrators, and researchers to measure, compare, and adjust for the importance of each procedure. Six indexes have been developed to quantify the risk of post-operative death after a range of surgeries (Table 1) [1-6]. Each of these indexes, 
Table 1 Summary of previous indexes predicting risk of death following surgery

\begin{tabular}{|c|c|c|c|}
\hline Index & Study Population ( $\mathrm{N}$ derivation/N validation) & Variables in model & $\begin{array}{l}\text { Validation } \\
\text { Area Under } \\
\text { the Curve }\end{array}$ \\
\hline $\begin{array}{l}\text { P-POSSUM } \\
{[6]}\end{array}$ & General surgical patients $(2500 / 7500)$ & $\begin{array}{l}\text { Age, cardiac history, respiratory history, blood pressure, pulse } \\
\text { rate, Glascow Coma Score, labs (hemoglobin, WBC, urea, Na+, } \\
\text { K+, ECG); operative severity, mulitple procedures, total blood } \\
\text { loss, peritoneal soiling, presence of malignancy, mode of } \\
\text { surgery }\end{array}$ & - \\
\hline $\mathrm{SRS}[5]$ & Patients of three general surgeons (3144/2780) & CEPOD classification; BUPA operative grade; ASA score & $\begin{array}{c}0.95(0.93 \\
0.97)[5]\end{array}$ \\
\hline $\begin{array}{l}\text { Cr-POSSUM } \\
{[1]} \\
\end{array}$ & $\begin{array}{l}\text { Patients having emergency or elective colorectal } \\
\text { surgery }(4079 / 2691)\end{array}$ & $\begin{array}{l}\text { Age; cardiac failure; SBP; pulse; urea; haemoglobin; operative } \\
\text { severity; peritoneal soiling; operative urgency; cancer staging }\end{array}$ & $\begin{array}{c}0.90(0.88 \\
0.92)[1]\end{array}$ \\
\hline $\begin{array}{c}\text { Nottingham } \\
{[3]}\end{array}$ & $\begin{array}{l}65+\text { years, acute or elective surgical and acute } \\
\text { urological patients undergoing operative or } \\
\text { conservative treatment }(2923 / 1362)\end{array}$ & $\begin{array}{c}\text { Age; white cell count; urea; pulse rate; mean systolic and } \\
\text { diastolic blood pressure; emergency admission; emergency } \\
\text { operation; major operation; vascular disease; malignant disease; } \\
\text { conservative treatment }\end{array}$ & $\begin{array}{c}0.86(0.82, \\
0.89)[3]\end{array}$ \\
\hline AFC $[25,26]$ & $\begin{array}{l}\text { Undergoing open or laparoscopic surgery for } \\
\text { colorectal cancers or diverticular disease (1421/395) }\end{array}$ & $\begin{array}{c}\text { Emergency surgery (surgery withing } 24 \text { hours of admission); } \\
\text { weight loss }>10 \% \text { within past } 6 \text { months; neurological disease } \\
\text { history; age }>70 \text { years }\end{array}$ & $0.89[4]$ \\
\hline $\begin{array}{l}\text { E-POSSUM } \\
{[2]}\end{array}$ & $\begin{array}{l}65+\text { years; undergoing first colorectal operation or } \\
\text { early colorectal reoperation (791/395) }\end{array}$ & $\begin{array}{c}\text { Age group (WHO classification); physiological score (cardiac } \\
\text { history, respiratory history, blood pressure, pulse rate, Glascow } \\
\text { Coma Score, haemoglobin level, white cell count, urea } \\
\text { concentration, Na+ level, K+ level, electrocardiography); } \\
\text { operative severity score (operative severity, mulitple } \\
\text { procedures, total blood loss, peritoneal soiling, presence of } \\
\text { malignancy, mode of surgery) }\end{array}$ & $\begin{array}{c}0.86(0.81 \\
0.92)[2]\end{array}$ \\
\hline
\end{tabular}

CEPOD - Confidential Enquiry into Perioperative Death

BUPA - British United Provident Association

ASA - American Society of Anesthesiologists

however, requires clinical information that is usually unavailable in routinely collected administrative data.

In this study, we derived and internally validated an index to measure the influence of a broad range of surgeries on in-hospital mortality. Our goal was to quantify the independent association of all procedures with the risk of death in hospital. To do this, we first grouped procedures based on administrative codes and the procedure's urgency status and then determined which of these procedure-urgency groups were associated with risk of death in hospital after adjusting for factors that are highly predictive of this outcome. We then created a scoring system to quantify the independent association of significant procedures with risk of death in hospital. This index can be calculated using administrative data and estimates the risk of death in hospital from these procedures that is independent of other factors associated with this outcome. It can be used to help risk-adjust analyses using administrative data that have death in hospital as an outcome. Such analyses could be done to identify factors independently associated with death in hospital and, in some situations, compare quality of care between institutions.

\section{Methods}

\section{Study Setting}

This study took place at The Ottawa Hospital (TOH), a tertiary-care teaching facility with three sites that averaged 20000 admissions annually during the study period. TOH functions within a publicly funded health care system. $\mathrm{TOH}$ is the sole regional provider of trauma care, thoracic surgery, and neurosurgical interventions, and provides most of the region's oncological care.

\section{Patients}

We included all admissions to the hospital (including same-day surgeries) between 1 April 2004 and 1 April 2009. "Same-day surgeries" included patients who had their surgery on the same day on which they were admitted to hospital. These patients were typically discharged home the same day but may have been kept in hospital if complications occurred or if additional monitoring was required. We started patient recruitment in April 2004 to ensure that our hospital had at least two years of experience coding procedures with the Canadian Classification of Interventions (CCI) coding system (which was introduced in April 2002). Patient recruitment ended in April 2009 (the last complete year of data available when the analyses were conducted). To apply the Kaiser Permanente In-patient Risk Adjustment Model (KP-IRAM) [7] - the method used to adjust for other risk factors associated with death in hospital - we excluded all patients with age $\leq 15$ years at admission, all delivery-related obstetrical admissions, and those 
who were transferred to or from $\mathrm{TOH}$. Throughout this study, the unit of analysis was the hospitalization.

\section{Candidate Procedures}

We used multiple binomial logistic regression to derive our index. We chose death in hospital as the model outcome because it is accurately recorded and is important to all potential users of the index. There were a total of 4013 hospital deaths (2.9\% of all admissions) in the derivation cohort. Our logistic model could therefore test a maximum of 400 procedures or surgeries (i.e. 10 deaths per exposure) to safely avoid problems with over-fitting and model instability [8].

We identified candidate procedures using their Canadian Classification of Interventions (CCI) code. The CCI system contains more than 18,000 unique codes. We therefore grouped procedures using the first five alphanumerics of each code (which identifies the anatomical area and the intervention type) and limited our study to therapeutic procedures (i.e. CCI section 1). We used the admission status of the hospitalization (i.e. elective vs. non-elective admission) to classify the procedure urgency since urgency is an important and independent predictor of post-procedural outcomes [9-14]. Procedures that could not be performed electively (such as cardiac resuscitation, implantation of an internal device in the thoracic descending aorta, and control of bleeding in the thoracic cavity) were classified as "non-elective" regardless of the admission status of the hospitalization.

There were 3984 unique procedure-urgency combinations during the study period. Since this exceeded the maximum number of variables allowed in our model without overfitting $(n=400)$, we used three filters to exclude procedures. First, we only included procedures that were conducted on the day of the principal procedure (defined as the procedure considered by the health records analyst to be most significant during the patient's hospital stay). In $5 \%$ of hospitalizations, coded procedures occurred on more than one day. In such cases, only procedures that occurred on the day of the principal procedure were considered. Second, procedures had to be conducted at least once per month at our hospital during the study period (independent of its urgency status). Finally, the p-value for the association of the procedure with death in hospital (after adjusting for risk of death in-hospital measured with KP-IRAM) had to be less than 0.5 .

\section{Adjusting for Risk of Death in Hospital}

To adjust for risk of death in hospital due to patient and hospitalization factors, we used the Kaiser Permanente In-patient Risk Adjustment Model (KP-IRAM) [7]. This model was derived and internally validated on almost 260,000 hospitalizations at 17 hospitals belonging to the
Kaiser Permanente Health Plan and was subsequently validated at our hospital [15]. The KP-IRAM includes six covariates including: patient age; patient sex; admission urgency (i.e. elective or emergent) and service (i.e. medical or surgical); admission diagnosis; severity of acute illness as measured by the Laboratory-based Acute Physiology Score (LAPS); and chronic comorbidities measured by the Comorbidity Point Score (COPS). Using the admission diagnosis, hospitalizations were grouped into "Primary Conditions," and a separate logistic regression model was created for each group. Interaction terms between age, LAPS, and comorbidity score were included. The model had excellent discrimination $(\mathrm{c}$-statistic $=0.88)$ and calibration ( $\mathrm{p}$-value of Hosmer Lemeshow statistic for the entire cohort was 0.66 ) for all-cause death in hospital.

We made three minor modifications to the KP-IRAM for this study. First, Canada switched from the International Classification of Diseases (ICD) 9-CM system (used in the KP-IRAM) to the ICD-10-CA system in 2002. We therefore used tables (provided by Canadian Institute for Health Information) to translate ICD-9-CM admission diagnoses to ICD-10-CA codes. Second, we measured chronic comorbidities using the Elixhauser Index [16] instead of the COPS because the KP-IRAM performed equally well using either comorbidity index [15]. Finally, the KP-IRAM was calculated on the day of the procedure (rather than at admission) for people who had one of the procedures included in the model. This model was used to estimate each patient's risk of death in hospital at the time of the procedure (expressed as a number that ranged between 0 and 1 ).

\section{Creation of the Procedural Index for Mortality Risk (PIMR) Score}

We randomly separated patients into equally sized derivation and validation groups. Using the derivation group, we ran a binary logistic regression model with death in hospital as the outcome and the KP-IRAM estimated risk as the adjusting covariate. The index day for patients undergoing one of the procedures considered for the model was the day of the procedure. For all other patients, the index day was the day of admission. Values of all covariates for the KP-IRAM model were those on the index day. We used stepwise variable selection to identify which candidate procedure-urgency combinations were independently associated with death in hospital. Surgeries with a 2 -sided p-value less than 0.05 were retained in the model.

We then used the methods described by Sullivan et. al. [17] to modify the parameter estimates of this regression model into an index. The number of points assigned to each procedure equaled its regression coefficient divided by the coefficient in the model with the 
smallest absolute value. We rounded this quotient to the nearest whole number. This number translated the parameter estimates into units relative to the procedure with the smallest, independently significant association with death in hospital. Therefore, the association of a procedure assigned two points was twice as important for predicting risk of death in hospital as a procedure with one point. Each person's total Procedural Independent Mortality Risk (PIMR) score was then calculated by summing up the points of all significant procedural groups for which they had been coded.

When calculating the PIMR score, we tallied up only those procedures that were performed on the index day (i.e. the day on which the principal procedure was conducted). Procedures done on other days did not influence the PIMR score. The PIMR score also did not capture whether or not the procedure was the first procedure conducted during the hospitalization.

\section{Assessment of the PIMR score}

In the validation group, we described the distribution of the PIMR score and used logistic regression to measure the association of the PIMR score alone with risk of death in hospital.

We then measured the influence of the PIMR score on risk of death in hospital independent of other factors associated with this outcome. "Discrimination" measures a model's ability to distinguish between patients who did and did not die in hospital and was measured using the c-statistic [18]. "Calibration" measures the accuracy of a model's predicted risk of death and was measured by dividing the study cohort into deciles and strata based on the estimated risk of death. Within each decile and stratum, observed and expected death rates were deemed similar if the $95 \%$ confidence interval around the former (calculated using exact methods [19]) included the latter. Overall calibration was summarized using the Hosmer Lemeshow statistic [20]. Table cells containing less than five observations were censored to maintain patient confidentiality.

In the validation group, we then compared the predictive performance of models containing the KP-IRAM with and without the PIMR score. To do this, we used two statistical measures: the Integrated Discrimination Improvement (IDI) [21] and the Net Reclassification Improvement (NRI) [22]. The IDI is the discrimination slope (the mean predicted risk in patients with the event minus that of patients without the event) of a model with the KP-IRAM and PIMR as independent predictors minus the discrimination slope of a model with the KPIRAM alone as the independent predictor. An IDI above zero indicates improved discrimination (i.e. a larger separation in mean predicted risk between events and nonevents) with the addition of the PIMR. The NRI represents the net proportion of correct reclassification (with correct reclassification defined as the predicted risk moving upwards for events and downwards for non-events) among events and non-events (calculated separately and then summed) when the predicted risk from the model with KP-IRAM and PIMR is compared to that from the model with KP-IRAM alone. We also calculated the net number of correct reclassifications when the PIMR was added to the KP-IRAM.

SAS 9.2 (Cary, NC) was used for all analyses. The study was approved by The Ottawa Hospital Research Ethics Board.

\section{Results}

There were 369588 admissions to The Ottawa Hospital between 1 April 2004 and 1 April 2009. 93971 of these hospitalizations were excluded from this study because patients were less than 15 years of age $(n=36820)$, patients were transferred from or to another hospital (n = 12 931), or admissions were obstetrical and deliveryrelated $(n=44220)$. We excluded another 157 admissions because they were missing a primary condition group (required to calculate the KP-IRAM). This left a total of 275460 hospital admissions (137 730 in both the derivation and the validation group) consisting of 172396 unique individuals. A description of patients in the derivation cohort is provided in Table 2 . The validation group did not differ significantly from the derivation group (see additional file 1).

In the entire cohort, a total of 1939 therapeutic procedures were coded during the study period. 1436 procedures were excluded because less than one procedure per month was performed during the study period. The remaining 503 procedures included a total of 938 procedure-urgency combinations. After adjusting for the Kaiser Permanente In-patient Risk Adjustment Model (KPIRAM) death risk estimate, the p-value of the association of 726 of these procedure-urgency combinations exceeded 0.5 in the derivation cohort and were therefore excluded. This left a total of 212 procedure-urgency combinations (including 168 individual surgeries) expressed as binomial (i.e. 1/0) variables that were offered to the logistic model (see additional file 2).

After adjusting for important patient and admission factors, 56 procedure-urgency combinations (comprising 52 individual procedures) were independently associated with death in hospital (Table 3). 37 emergent and eight elective procedures were independently associated with an increased risk of death in hospital, while four emergent and seven elective procedures were protective. In the validation set, there were 22664 (16.4\%) admissions where the patient underwent at least one PIMR procedure, with $83 \%$ of these procedures occurring within the first three days of the hospitalization. Procedures having 
Table 2 Description of study hospitalizations in derivation cohort

\begin{tabular}{|c|c|c|c|}
\hline & $\begin{array}{l}\text { Entire Cohort }(n=137 \\
730)\end{array}$ & $\begin{array}{l}\text { Died in Hospital }(n=4 \\
\text { 013) }\end{array}$ & $\begin{array}{l}\text { Discharged Alive }(n=133 \\
\text { 717) }\end{array}$ \\
\hline Mean age (SD) & $58.9(18.4)$ & $72.8(14.6)$ & $58.5(18.3)$ \\
\hline Female, n (\%) & $71724(52.1)$ & $18.7(46.5)$ & $69856(52.2)$ \\
\hline Urgent admission, n (\%) & $54400(39.5)$ & $3824(95.3)$ & $50576(37.8)$ \\
\hline Surgical service, $\mathrm{n}(\%)$ & $89586(65.0)$ & $821(20.5)$ & $88765(66.4)$ \\
\hline Median Elixhauser score ${ }^{16}$ (IQR) & $0(0-4)$ & $10(4-16)$ & $0(0-3)$ \\
\hline Mean LAPS at admission* (SD) & $11.6(22.3)$ & $52.4(32.9)$ & $10.4(20.7)$ \\
\hline Median risk of death at admission $(\mathrm{IQR})^{* *}$ & $0.0011(0.0001-0.0135)$ & $0.1896(0.0869-0.3517)$ & $0.0009(0.0001-0.0109)$ \\
\hline \multicolumn{4}{|l|}{ Most Common Procedures, n (\%) } \\
\hline Lens excision & $18571(13.5 \%)$ & $0(0.0 \%)$ & $18571(13.9 \%)$ \\
\hline Angioplasty & 4177 (3.0\%) & $68(1.7 \%)$ & 4109 (3.1\%) \\
\hline Pharmacotherapy, total body & $3652(2.7 \%)$ & $93(2.3 \%)$ & $3559(2.7 \%)$ \\
\hline Respiratory ventilation & $3236(2.3 \%)$ & $682(17.0 \%)$ & $2554(1.9 \%)$ \\
\hline Repair, muscles of the chest and abdomen & $3169(2.3 \%)$ & $24(0.6 \%)$ & $3145(2.4 \%)$ \\
\hline Partial hysterectomy & $2628(1.9 \%)$ & $0(0.0 \%)$ & $2628(2.0 \%)$ \\
\hline $\begin{array}{l}\text { Installation of external appliance, circulatory system } \\
\text { NEC }\end{array}$ & $2514(1.8 \%)$ & $104(2.6 \%)$ & $2410(1.8 \%)$ \\
\hline Total excision of vitreous & $2385(1.7 \%)$ & $0(0.0 \%)$ & $2385(1.8 \%)$ \\
\hline Pharmacotherapy (local), vessels of heart & $2182(1.6 \%)$ & $26(0.6 \%)$ & $2156(1.6 \%)$ \\
\hline Total hysterectomy & $2211-5(1.6 \%)$ & $<=5(0.0 \%)$ & $2210(1.7 \%)$ \\
\hline
\end{tabular}

the strongest association with death in hospital included cardiac resuscitation, ventriculectomy, pericardial drainage, and pelvic irradiation. A full description of each procedure that was independently associated with death in hospital is given in Additional File 3.

Four procedures were independently associated with risk of death in hospital regardless of whether the procedure was done emergently or electively (Table 4). In two cases, the elective version of the procedure was assigned more points (indicating a higher risk of death in hospital) than the emergent version of the procedure.

Parameter estimates for procedures in the final logistic model were modified into the Procedural Index for Mortality Risk (PIMR) score (Table 3 ). The PIMR score for individual procedures ranged from -7 to +11 . Since $84 \%$ of admissions had none of the included procedures, most hospitalizations had a total PIMR score of 0 (Figure 1, left axis). The risk of death in hospital was significantly associated with the PIMR score (Figure 1, right axis). By itself, the PIMR score was moderately discriminative for death in hospital (c-statistic 67.3\%, 95\% CI 66.6\%-68.0\%).

The total PIMR score significantly changed the expected risk of death in hospital beyond that estimated by the KP-IRAM (Figure 2). The total PIMR score also significantly improved the ability to predict risk of inhospital death beyond that generated by the KP-IRAM. Model discrimination improved, as indicated by the cstatistic (increased from 0.929 [95\% CI 0.926-0.932] to 0.938 [0.935-0.941]) and the Integrated Discrimination Improvement (IDI) (0.04327, 95\% CI 0.0384-0.0482; $p<$ .0001). Model calibration (Figure 3) did not change (Hosmer-Lemeshow fit statistic decreased from 37.56 to 36.51). The Net Reclassification Improvement (NRI) analysis showed that although the overall net proportion of correct reclassification was negative $(-18.4 \%)$, the overall net number of correct reclassifications was positive $(+17923$ or $13 \%$ of the entire cohort, Table 5$)$.

\section{Discussion}

We derived and internally validated an index that used administrative data to quantify the relative contribution of a broad range of therapeutic procedures on the risk of death in hospital. We identified 52 procedures which (after adjusting for a robust and validated hospital mortality model) were significantly associated with the risk of death in hospital. We modified this model into an index that reflects the independent contribution of each procedure to the risk of death in hospital. By itself, and when added to an accurate model to predict hospital 
Table 3 Procedures independently associated with death in hospital

\begin{tabular}{|c|c|c|c|c|}
\hline Variable & Para-meter Estimate & Adjusted Odds Ratio & $(95 \% \mathrm{Cl})$ & PIMR Score \\
\hline Predicted risk of death* & 1.03 & 2.79 & $2.73-2.86$ & - \\
\hline \multicolumn{5}{|l|}{ Emergent Procedures } \\
\hline Resuscitation, heart NEC & 4.26 & 70.72 & $41.04-121.84$ & 11 \\
\hline Excision partial, ventricle & 3.71 & 40.91 & $5.03-333.05$ & 10 \\
\hline Repair, aortic valve & 2.91 & 18.27 & $4.90-68.19$ & 8 \\
\hline Immobilization, shoulder joint & 2.95 & 19.18 & $1.55-236.57$ & 8 \\
\hline Repair, patella & 2.81 & 16.60 & $1.61-171.01$ & 7 \\
\hline Repair, tricuspid valve & 2.38 & 10.85 & $1.77-66.34$ & 6 \\
\hline Implantation of device, descending thoracic aorta & 2.28 & 9.82 & $5.73-16.84$ & 6 \\
\hline Occlusion, abdominal arteries NEC & 2.44 & 11.53 & $4.67-28.49$ & 6 \\
\hline Implantation of internal device, abdominal cavity & 2.43 & 11.33 & $2.21-58.17$ & 6 \\
\hline Excision partial soft tissue, chest and abdomen & 2.46 & 11.76 & $2.38-58.01$ & 6 \\
\hline Drainage, ventricles of brain & 1.88 & 6.54 & $3.26-13.11$ & 5 \\
\hline Drainage, bronchus NEC & 1.77 & 5.89 & $1.33-26.20$ & 5 \\
\hline Ventilation, respiratory system NEC & 1.89 & 6.65 & $5.73-7.71$ & 5 \\
\hline Installation of external appliance, heart NEC & 2.00 & 7.38 & $2.72-20.01$ & 5 \\
\hline Stimulation, heart NEC & 1.54 & 4.68 & $2.89-7.56$ & 4 \\
\hline Extraction, arteries of leg NEC & 1.47 & 4.34 & $2.38-7.93$ & 4 \\
\hline Bypass, small intestine & 1.49 & 4.43 & $1.95-10.04$ & 4 \\
\hline Repair, small intestine & 1.35 & 3.86 & $1.91-7.80$ & 4 \\
\hline Drainage, meninges and dura mater of brain & 1.23 & 3.43 & $2.15-5.48$ & 3 \\
\hline Excision partial, brain & 1.33 & 3.77 & $1.98-7.20$ & 3 \\
\hline Control of bleeding, thoracic cavity NEC & 1.06 & 2.90 & $1.37-6.12$ & 3 \\
\hline Drainage, pericardium & 1.01 & 2.74 & $1.18-6.37$ & 3 \\
\hline Occlusion, vena cava (superior and inferior) & 1.25 & 3.50 & $1.60-7.65$ & 3 \\
\hline Control of bleeding, esophagus & 1.14 & 3.12 & $1.46-6.71$ & 3 \\
\hline Dilation, esophagus & 1.09 & 2.98 & $1.18-7.54$ & 3 \\
\hline Control of bleeding, small and large intestine & 1.10 & 3.00 & $1.22-7.38$ & 3 \\
\hline Amputation, tibia and fibula & 1.03 & 2.80 & $1.27-6.21$ & 3 \\
\hline Bypass with exteriorization, trachea & 0.58 & 1.78 & $1.12-2.84$ & 2 \\
\hline Implantation of internal device, stomach & 0.61 & 1.84 & $1.25-2.70$ & 2 \\
\hline Excision partial, small intestine & 0.78 & 2.18 & $1.33-3.58$ & 2 \\
\hline Excision partial, large intestine & 0.58 & 1.79 & $1.20-2.66$ & 2 \\
\hline Drainage, abdominal cavity & 0.67 & 1.96 & $1.43-2.69$ & 2 \\
\hline Implantation of internal device, hip joint & 0.82 & 2.26 & $1.59-3.22$ & 2 \\
\hline Fixation, femur & 0.76 & 2.13 & $1.52-2.99$ & 2 \\
\hline Amputation, femur & 0.92 & 2.51 & $1.20-5.24$ & 2 \\
\hline Drainage, pleura & 0.56 & 1.74 & $1.36-2.24$ & 1 \\
\hline Implantation of internal device, vena cava & 0.38 & 1.46 & $1.20-1.78$ & 1 \\
\hline Pharmacotherapy (local), vessels of heart & -0.68 & 0.51 & $0.31-0.85$ & -2 \\
\hline Excision total, appendix & -1.13 & 0.32 & $0.12-0.88$ & -3 \\
\hline Installation of external appliance, circulatory system NEC & -1.39 & 0.25 & $0.17-0.36$ & -4 \\
\hline Excision partial, abdominal cavity & -2.35 & 0.10 & $0.01-0.72$ & -6 \\
\hline \multicolumn{5}{|l|}{ Elective Procedures } \\
\hline Drainage, pericardium & 3.37 & 29.16 & $6.28-135.28$ & 9 \\
\hline Radiation, pelvis & 3.35 & 28.47 & $2.58-314.61$ & 9 \\
\hline Destruction, skin of abdomen and trunk & 2.75 & 15.61 & $1.89-128.67$ & 7 \\
\hline
\end{tabular}


Table 3 Procedures independently associated with death in hospital (Continued)

\begin{tabular}{llll}
\hline Excision partial, cerebellum & 2.17 & 8.75 & $2.01-38.11$ \\
\hline Pharmacotherapy, circulatory system NEC & 2.29 & 9.92 & $2.26-43.51$ \\
\hline Repair, abdominal arteries NEC & 1.75 & 5.77 & $1.36-24.54$ \\
\hline Amputation, femur & 1.92 & 6.81 & $1.93-24.01$ \\
\hline Ventilation, respiratory system NEC & 0.83 & 2.28 & $1.66-3.14$ \\
\hline Dilation, coronary arteries & -1.61 & 0.20 & $0.06-0.69$ \\
\hline Implantation of internal device, hip joint & -1.57 & 0.21 & $0.07-0.65$ \\
\hline Implantation of internal device, knee joint & -1.58 & 0.21 & $0.08-0.55$ \\
\hline Excision total, ovary with fallopian tube & -2.07 & 0.13 & -4 \\
\hline Repair, muscles of the chest and abdomen & -2.00 & $0.02-0.91$ \\
\hline Excision partial, prostate & -2.24 & -4 & $0.05-0.36$ \\
\hline Excision total, uterus and surrounding structures & -2.51 & 0.11 & $0.01-0.76$ \\
\hline
\end{tabular}

*The adjusted odds ratio represents the increased risk for every 10\% increase in death risk from the KP in-patient risk model.

See Additional File 3 for codes and a full description of each procedure (along with all component procedures).

NEC - Not elsewhere classified

mortality, the total Procedural Index for Mortality Risk (PIMR) score significantly predicted risk of death in hospital.

The importance of surgical interventions on hospital outcomes is reflected by the large number of indexes that use patient and hospitalization factors to predict the risk of post-procedural death (Table 1) [1-6]. The clinical variables in these indexes, along with their simplicity, increase their face validity to practicing clinicians. However, these clinical variables prohibit calculation of these indexes using administrative data. To develop our index, we started with a validated, highly accurate model to predict hospital mortality risk in all hospital patients. We then determined the risk of death after a broad range of procedures independent of that predicted from the KP-IRAM. Both by itself and when added to the KP-IRAM model, the PIMR was significantly associated with the risk of death in hospital.

The PIMR would primarily be used in analyses involving administrative data. Expressing this risk as a simple score facilitates our understanding of the relative importance of various interventions on death in hospital. When combined with the KP-IRAM, the PIMR had excellent discrimination and calibration for predicting risk of death in hospital. It is notable that the discrimination achieved with the KP-IRAM and PIMR was similar to that achieved using clinical based models (Table 1). The PIMR will allow researchers and administrators to gauge patient and procedural complexity of individual surgeons, services, or hospitals for descriptive or comparative purposes and will also let analysts adjust for the influence of a large range of therapeutic procedures on risk of hospital mortality.

The independent association between many of the PIMR procedures and risk of hospital death may reflect unresolved confounding of patient or hospitalization factors. The significance of several procedures (e.g. cardiac resuscitation) is likely due to important clinical events (e.g. cardiac arrest) that are identified by the procedure code and are not captured by the KP-IRAM. Further work is required to determine how much mortality risk is due to the procedure and how much is due to other underlying patient factors.

The addition of the PIMR to the KP-IRAM model significantly improved the ability to predict hospital mortality. The absolute increase of the model's c-statistic was small $(0.009$ or $0.9 \%)$. Several studies have shown that the overall, sequential improvement of model performance decreases as more and more variables are added $[23,24]$. However, the c-statistic of the KP-IRAM was already very high without the PIMR score (92.9\%). With the PIMR, the C-statistic increased more than $10 \%$ of the distance between the KP-IRAM and perfect discrimination. This indicates, along with the results presented in Figure 1, the strength of PIMR to predict risk

Table 4 Procedures independently associated with risk of death in-hospital regardless of procedure urgency

\begin{tabular}{lcc}
\hline Procedural Description & Points for emergent procedure & Points for elective procedure \\
\hline Ventilation, respiratory system NEC & 5 & 2 \\
Drainage, pericardium & 3 & 9 \\
Implantation of internal device, hip joint & 2 & -4 \\
Amputation, femur & 2 & 5 \\
\hline
\end{tabular}




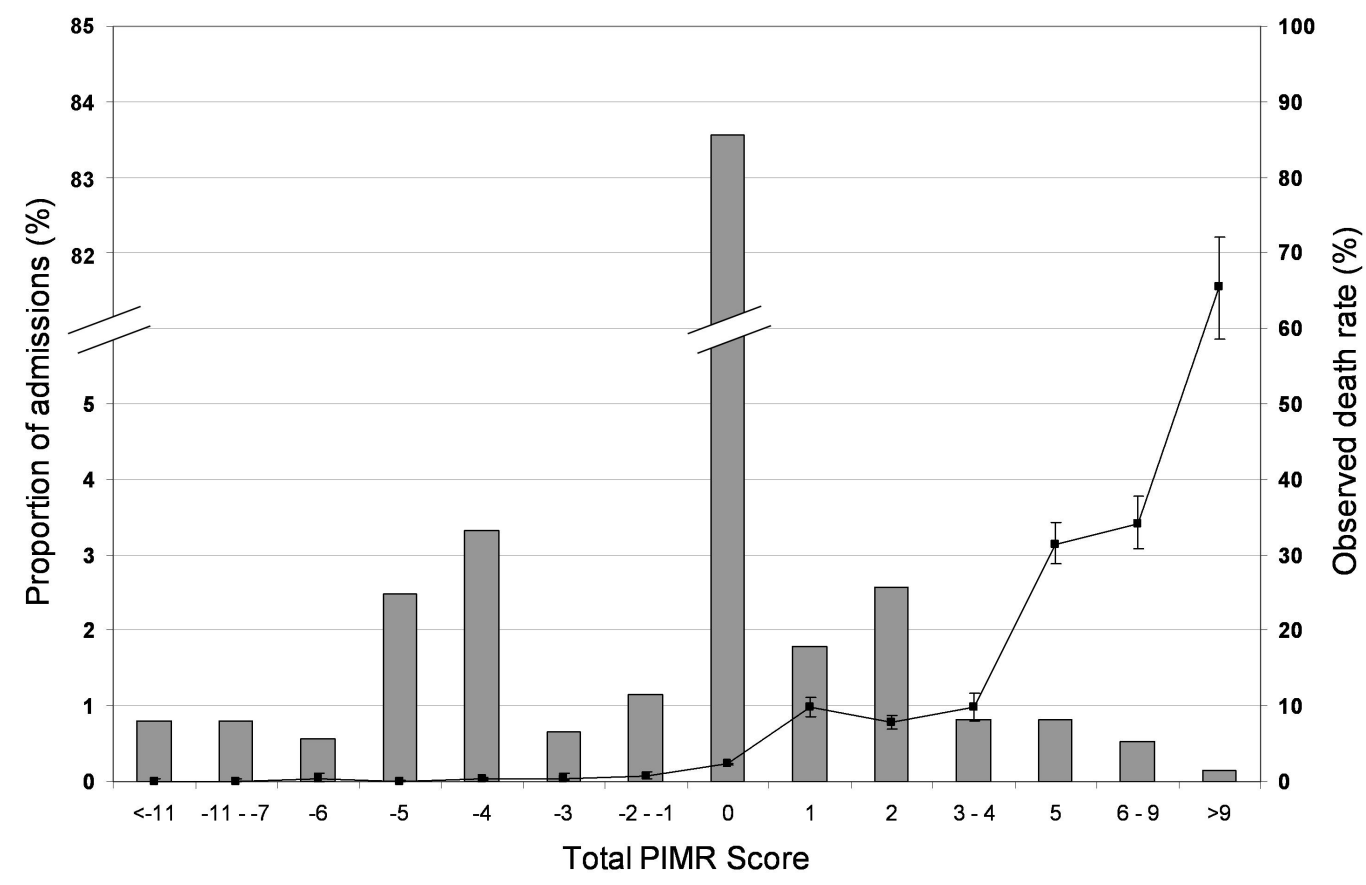

Figure 1 Frequency distribution of the total Procedural Index for Mortality Risk (PIMR) score among validation admissions. The horizontal axis presents the total PIMR score. The bars and left vertical axis presents the percent of hospitalizations with each total PIMR score. Individual PIMR scores were grouped to contain at least $0.5 \%$ of all admissions. The line and right vertical axis presents the observed number of deaths (with 95\% confidence intervals) in each PIMR score.

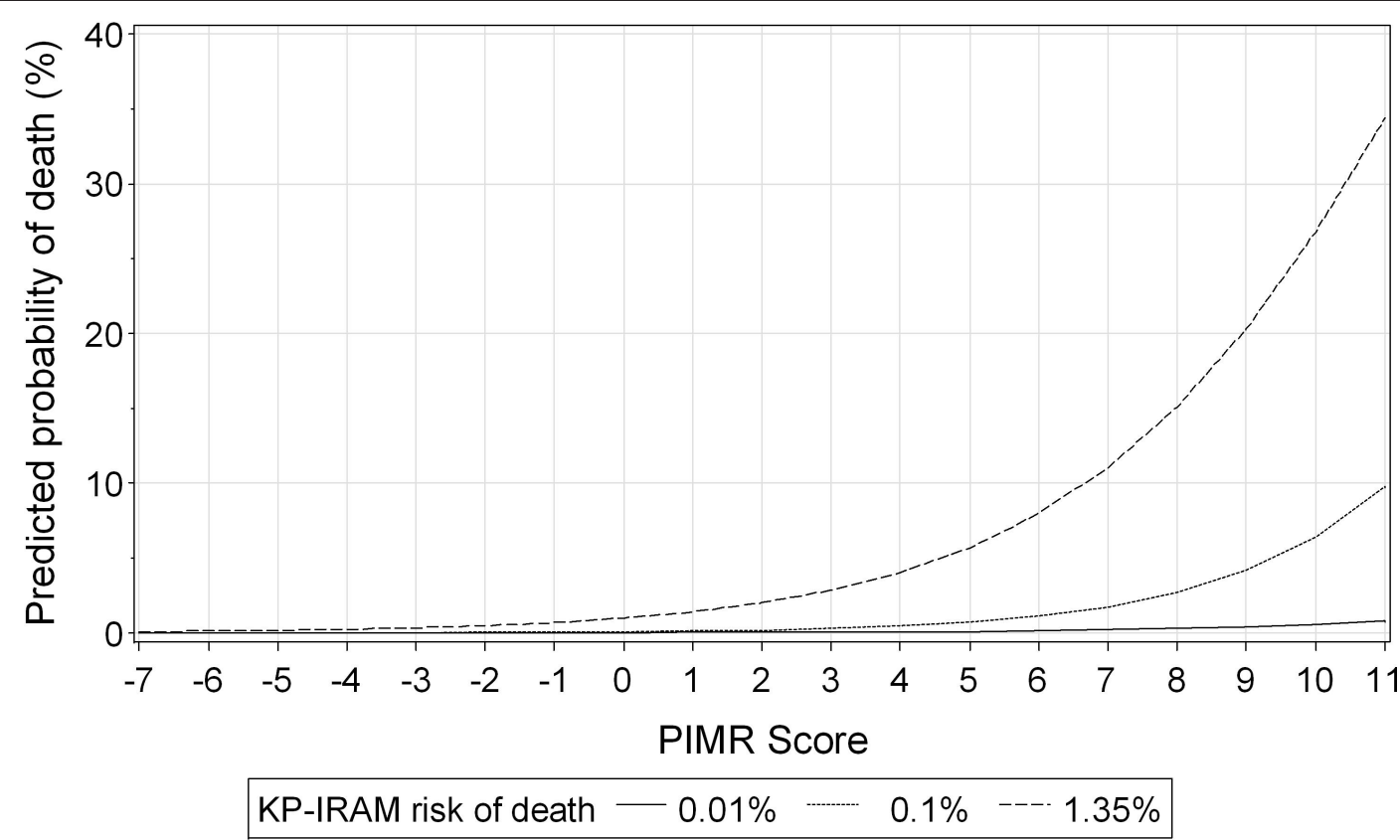

Figure 2 Effect of adding the Procedural Index for Mortality Risk (PIMR) score to the Kaiser Permanente Inpatient Risk Adjustment Methodology (KP-IRAM) on predicted risk of death in hospital. This graph presents the expected probability of death in hospital (vertical axis) for varying PIMR scores (horizontal axis). Risks are presented for people whose expected risk of death in hospital (based on the KP-IRAM) was at the $25^{\text {th }}$ percentile $\left(0.01 \%\right.$, solid line), $50^{\text {th }}$ percentile $\left(0.11 \%\right.$, long-dashed line), and $75^{\text {th }}$ percentile $(1.35 \%$, short-dashed line). 

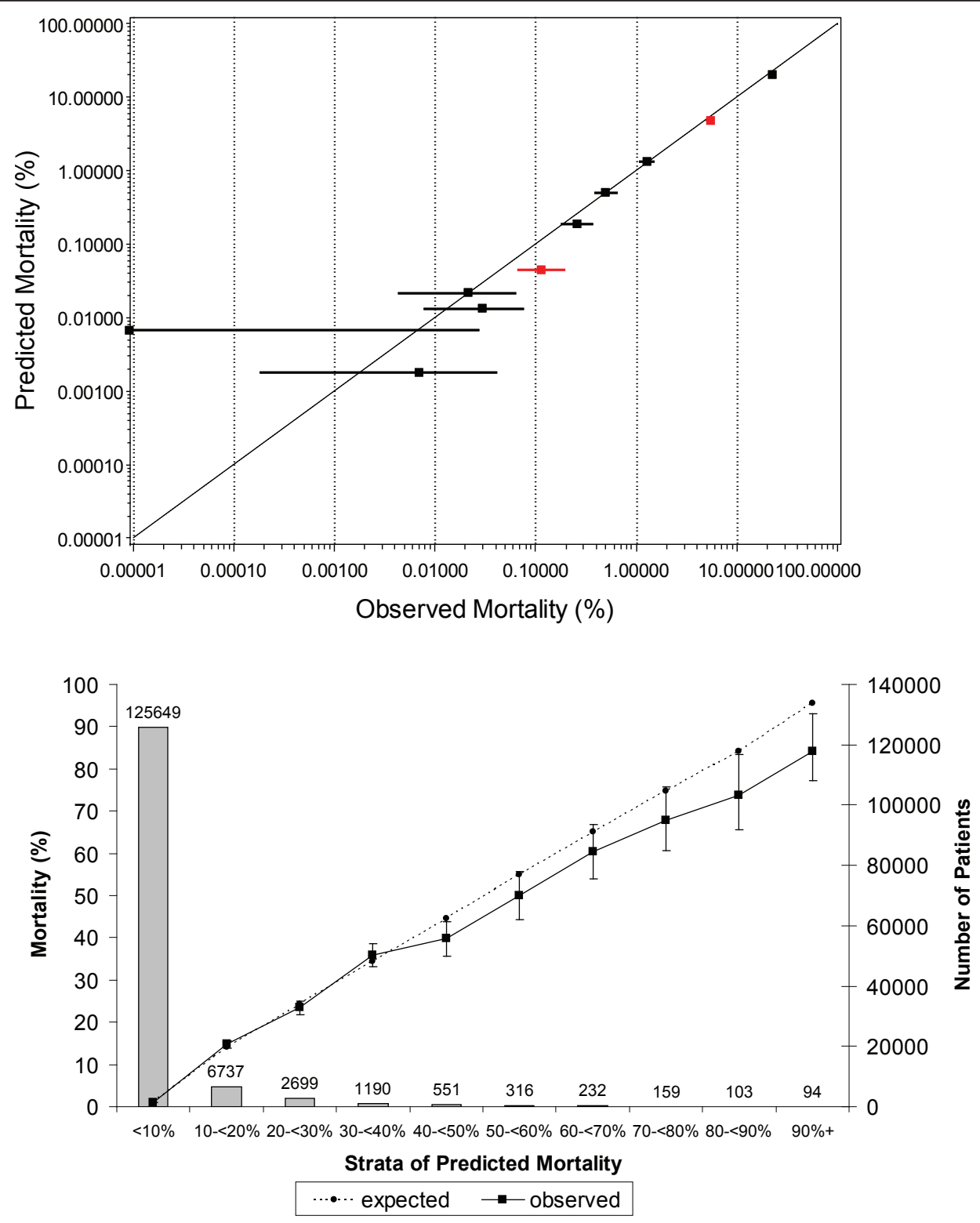

Figure 3 Calibration of KP-IRAM and PIMR to predict death in hospital. These figures compare observed and expected death rates when the validation group was divided into expected risk deciles (top) and strata (bottom). The decile plot presents observed mortality rates with $95 \%$ confidence intervals with those in red significantly differing from expected.

Table 5 Results of the Net Reclassification Improvement (NRI) analysis:

\begin{tabular}{|c|c|c|c|c|c|c|}
\hline \multirow[t]{2}{*}{ Discharge status (N) } & \multicolumn{2}{|c|}{ Correct reclassification* } & \multicolumn{2}{|c|}{ Incorrect reclassification $^{+}$} & \multicolumn{2}{|c|}{ Net correct reclassification ${ }^{\ddagger}$} \\
\hline & $\%$ & $\mathrm{~N}$ & $\%$ & $\mathrm{~N}$ & $\%$ & $\mathrm{~N}$ \\
\hline Dead (4040) & 33.6 & 1357 & 66.4 & 2683 & -32.8 & -1325 \\
\hline Alive (133690) & 57.2 & 76471 & 42.8 & 57219 & +14.4 & +19251 \\
\hline \multicolumn{3}{|c|}{ Overall net correct reclassification } & & & $\begin{array}{l}-18.4 \\
\end{array}$ & +17926 \\
\hline
\end{tabular}

*Correct reclassification: 'up' for events and 'down' for non-events, where 'up' is a higher predicted risk of death from the new vs old model and 'down' is a lower predicted risk of death from the new vs old model

'Incorrect reclassification: 'down' for events and 'up' for non-events

${ }^{\ddagger}$ Net correct reclassification: correct reclassification - incorrect reclassification 
of death with or without other covariates associated with death risk in hospital.

We believe that two steps could greatly improve the PIMR. The PIMR relies on procedure codes whose accuracy has not been validated. Our study's objective was to derive and validate an index that determines the independent influence of various procedures on hospital mortality. Strictly speaking, however, the PIMR measures the independent influence of codes for various procedures - rather than the procedures themselves - on hospital mortality. Without knowing the accuracy of each code for its respective procedure, we are uncertain how strong a surrogate each code is for the actual procedure. Before one uses the PIMR for individual patient risk prediction, the accuracy of the procedure codes contained in the PIMR should be validated.

The second major limitation of the PIMR is its imputation of procedural urgency using admission urgency status. For most hospitalizations, admission and procedural urgency will be identical but situations could arise in which they would differ. For example, consider a patient admitted electively for a hip replacement who has an acute myocardial infarction requiring an emergent angioplasty. In this case, the angioplasty urgency would be misclassified as an elective procedure. We believe that this bias explains why the number of points assigned to two elective procedures exceeded that for their emergent counterpart (Table 4). The PIMR could be improved by using an accurate classification of procedural urgency.

There are other limitations to the PIMR. First, the PIMR requires that surgical procedures are coded using the Canadian Classification of Interventions (CCI). Without validated translation tables to other procedural coding systems, this limits its use to Canadian institutions. Second, the PIMR was derived and validated in a single hospital. While objective and universal criteria are used to code procedures, it is possible that local coding practices could change the PIMR's validity in other patient populations. Third, most procedures are not included in the PIMR because they were not independently associated with risk of death in hospital. As a result, the PIMR should be used as an adjunct to other factors associated with risk of death in hospital - such as those in the KP-IRAM to compare outcomes after various surgeries. Researchers should exercise some caution if this index is used when inferring institutional quality of care issues using hospital mortality. Some of the components in the PIMR (such as heart resuscitation) could result from poor quality of care, the adjustment of which could hide such problems.

Finally, our analyses did not include surgeries that were infrequently conducted at our hospital.

\section{Conclusion}

We have derived and internally validated an index to express the independent association of a broad range of procedures with risk of death in hospital. When this is added to a validated hospital death risk index, we can accurately predict post-procedural risk of death.

\section{Additional material}

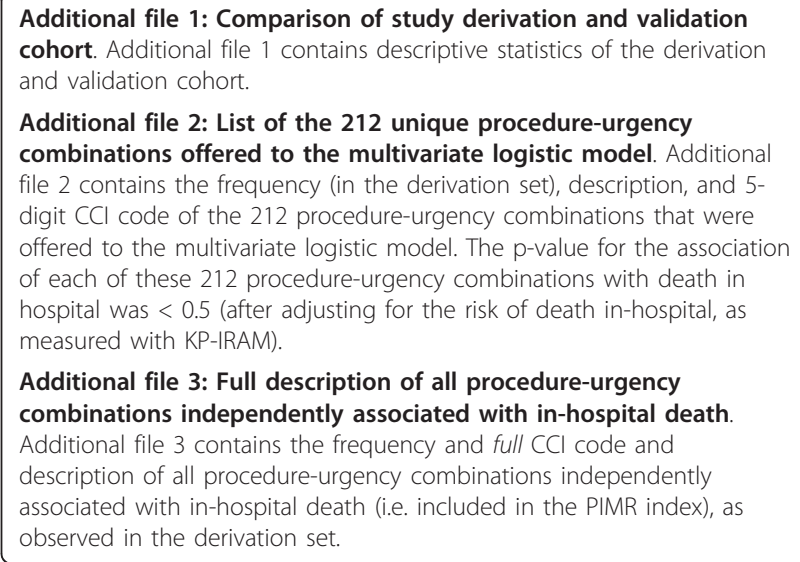

Additional file 2: List of the $\mathbf{2 1 2}$ unique procedure-urgency combinations offered to the multivariate logistic model. Additional file 2 contains the frequency (in the derivation set), description, and 5 digit $\mathrm{CCl}$ code of the 212 procedure-urgency combinations that were offered to the multivariate logistic model. The $p$-value for the association of each of these 212 procedure-urgency combinations with death in hospital was $<0.5$ (after adjusting for the risk of death in-hospital, as measured with KP-IRAM).

Additional file 3: Full description of all procedure-urgency combinations independently associated with in-hospital death. Additional file 3 contains the frequency and full $\mathrm{CCl}$ code and description of all procedure-urgency combinations independently associated with in-hospital death (i.e. included in the PIMR index), as observed in the derivation set.

\section{Acknowledgements}

This study was supported by the Department of Medicine, University of Ottawa.

\section{Author details}

${ }^{1}$ Clinical Epidemiology Program, Ottawa Hospital Research Institute, 1053 Carling Avenue, Ottawa, K1Y 4E9, Canada. ${ }^{2}$ Institute for Clinical Evaluative Sciences, 1053 Carling Avenue, Ottawa, K1Y 4E9, Canada. ${ }^{3}$ Department of Medicine, University of Ottawa, 1053 Carling Avenue, Ottawa, K1Y 4E9, Canada.

\section{Authors' contributions}

CVW conceived of the study; directed the study design and statistical analysis; and drafted the manuscript. JW participated in the study design; performed the statistical analysis; created the tables, additional files, and figures; and edited the manuscript. CB performed the literature search and extracted the information in Table 1. AF provided the study data, participated in the study design, and reviewed the manuscript for important clinical and intellectual content. All authors have read and approved the final manuscript.

\section{Competing interests}

The authors declare that they have no competing interests.

Received: 25 March 2011 Accepted: 7 October 2011

Published: 7 October 2011

\section{References}

1. Tekkis PP, Prytherch DR, Kocher HM, Senapati A, Poloniecki JD, Stamatakis JD, Windsor AC: Development of a dedicated risk-adjustment scoring system for colorectal surgery (colorectal POSSUM). British Journal of Surgery 2004, 91:1174-82.

2. Tran Ba LP, du Montcel ST, Duron JJ, Levard H, Suc B, Descottes B, Desrousseaux B, Hay JM: Elderly POSSUM, a dedicated score for prediction of mortality and morbidity after major colorectal surgery in older patients. British Journal of Surgery 2010, 97:396-403.

3. Lloyd H, Ahmed I, Taylor S, Blake JR: Index for predicting mortality in elderly surgical patients. British Journal of Surgery 2005, 92:487-92. 
4. Alves A, Panis Y, Mantion G, Slim K, Kwiatkowski F, Vicaut E: The AFC score: validation of a 4-item predicting score of postoperative mortality after colorectal resection for cancer or diverticulitis: results of a prospective multicenter study in 1049 patients. Annals of Surgery 2007, 246:91-6.

5. Sutton R, Bann S, Brooks M, Sarin S: The Surgical Risk Scale as an improved tool for risk-adjusted analysis in comparative surgical audit. British Journal of Surgery 2002, 89:763-8.

6. Prytherch DR, Whiteley MS, Higgins B, Weaver PC, Prout WG, Powell SJ: POSSUM and Portsmouth POSSUM for predicting mortality. Physiological and Operative Severity Score for the enUmeration of Mortality and morbidity. British Journal of Surgery 1998, 85:1217-20.

7. Escobar GJ, Greene JD, Scheirer P, Gardner MN, Draper D, Kipnis P: Riskadjusting hospital inpatient mortality using automated inpatient, outpatient, and laboratory databases. Med Care 2008, 46:232-9.

8. Harrell FE: General aspects of fitting regression models. Regression Modelling Strategies New York: Springer; 2001, 16-24.

9. Aust JB, Henderson W, Khuri S, Page CP: The impact of operative complexity on patient risk factors. Annals of Surgery 2005, 241:1024-7.

10. Shah KB, Kleinman BS, Rao TL, Jacobs HK, Mestan K, Schaafsma M: Angina and other risk factors in patients with cardiac diseases undergoing noncardiac operations. Anesthesia \& Analgesia 1990, 70:240-7.

11. Detsky AS, Abrams HB, McLaughlin JR, Drucker DJ, Sasson Z, Johnston N, Scott JG, Forbath N, Hilliard JR: Predicting cardiac complications in patients undergoing non-cardiac surgery. J Gen Intern Med 1986, 1:211-9.

12. Goldman L, Caldera DL, Nussbaum SR, Southwick FS, Krogstad D, Murray B, Burke DS, O'Malley TA, Goroll AH, Caplan CH: Multifactorial index of cardiac risk in noncardiac surgical procedures. N Engl J Med 1977, 297:845-50.

13. Kumar R, McKinney WP, Raj G, Heudebert GR, Heller HJ, Koetting M, McIntire DD: Adverse cardiac events after surgery: assessing risk in a veteran population. J Gen Intern Med 2001, 16:507-18.

14. Larsen SF, Olesen $\mathrm{KH}$, Jacobsen $\mathrm{E}$, Nielsen $\mathrm{H}$, Nielsen AL, Pietersen A, Jensen OJ, Pedersen F, Waaben J, Kehlet $\mathrm{H}$ : Prediction of cardiac risk in non-cardiac surgery. European Heart Journal 1987, 8:179-85.

15. van Walraven C, Escobar GJ, Greene JD, Forster AJ: The Kaiser Permanente inpatient risk adjustment methodology was valid in an external patient population. Journal of Clinical Epidemiology 2010, 63:798-803.

16. van Walraven $C$, Austin $P C$, Jennings $A$, Quan $H$, Forster AJ: A modification of the Elixhauser comorbidity measures into a point system for hospital death using administrative data. Med Care 2009, 47:626-33.

17. Sullivan LM, Massaro JM, D'Agostino RB Sr: Presentation of multivariate data for clinical use: The Framingham Study risk score functions. Stat Med 2004, 23:1631-60.

18. Gonen M: Single Continuous Predictor. Analyzing receiver operating characteristic curves with SAS Cary, N.C.: SAS Institute Inc; 2007, 15-36.

19. Fleiss $J$, Levin B, Paik MC: Poisson Regression. Statistical Methods for Rates and Proportions Hoboken: John Wiley \& Sons; 2003, 340-72

20. Hosmer DW, Hosmer T, Le Cessie S, Lemeshow S: A comparison of goodness-of-fit tests for the logistic regression model. Stat Med 1997, 16:965-80.

21. Pencina MJ, D'Agostino RB Sr, D'Agostino RB Jr, Vasan RS: Evaluating the added predictive ability of a new marker: from area under the ROC curve to reclassification and beyond. Stat Med 2008, 27:157-172.

22. Pencina MJ, D'Agostino RB Sr, Steyerberg EW: Extensions of net reclassification improvement calculations to measure usefulness of new biomarkers. Stat Med 2011, 30:11-21.

23. Pine $M$, Jordan HS, Elixhauser A, Fry DE, Hoaglin DC, Jones B, Meimban R, Warner D, Gonzales J: Enhancement of Claims Data to Improve Risk Adjustment of Hospital Mortality. JAMA: The Journal of the American Medical Association 2007, 297:71-6.

24. Tabak YP, Sun X, Derby KG, Kurtz SG, Johannes RS: Development and Validation of a Disease-Specific Risk Adjustment System Using Automated Clinical Data. Health Serv Res 2010, 45:1815-35.

25. Slim K, Panis Y, Alves A, Kwiatkowski F, Mathieu P, Mantion G, Association Française de Chirurgie: Predicting postoperative mortality in patients undergoing colorectal surgery. World Journal of Surgery 2006, 30:100-6.

26. Alves A, Panis Y, Mathieu P, Mantion G, Kwiatkowski F, Slim K, Association Française de Chirurgie: Postoperative mortality and morbidity in French patients undergoing colorectal surgery: results of a prospective multicenter study. Archives of Surgery 2005, 140:278-83.

\section{Pre-publication history}

The pre-publication history for this paper can be accessed here: http://www.biomedcentral.com/1472-6963/11/258/prepub

doi:10.1186/1472-6963-11-258

Cite this article as: van Walraven et al:: The Procedural Index for Mortality Risk (PIMR): an index calculated using administrative data to quantify the independent influence of procedures on risk of hospital death. BMC Health Services Research 2011 11:258.

\section{Submit your next manuscript to BioMed Central and take full advantage of:}

- Convenient online submission

- Thorough peer review

- No space constraints or color figure charges

- Immediate publication on acceptance

- Inclusion in PubMed, CAS, Scopus and Google Scholar

- Research which is freely available for redistribution

Submit your manuscript at www.biomedcentral.com/submit
Biomed Central 